\title{
EXTERNALIZACIÓN DE LA GESTIÓN DEL CONOCIMIENTO COMO CLAVE PARA LA COMPETITIVIDAD DEL SECTOR BIOTECNOLÓGICO COLOMBIANO*
}

\author{
Recibido: 25 de marzo de 2014 Aprobado 18 de agosto 2015 \\ Guillermo Rodrigo Corredor Castellanos ${ }^{* *}$
}

\section{RESUMEN}

Colombia, como país megadiverso, cuenta con importantes ventajas competitivas en el mercado global de materias primas e ingredientes naturales esenciales para un sinnúmero de actividades industriales. Sin embrago, las prácticas actuales relacionadas con la provisión de ingredientes naturales derivados de la biodiversidad ponen de presente las divergencias en cuanto al alcance de las normas sobre transferencia de tecnología contenidas en varios instrumentos multilaterales. El presente documento aborda el concepto de externalización de la gestión del conocimiento dentro del contexto de las políticas públicas orientadas al uso comercial de los recursos de la biodiversidad, indagando por las condiciones ideales que pueden favorecer la deslocalización de actividades de investigación y desarrollo por parte de corporaciones multinacionales respecto de la bioprospección e investigación fundamental, para que de esta manera los recursos de la biodiversidad puedan ser objeto de actividades de valor agregado dentro del territorio nacional.

\section{PALABRAS CLAVE}

Outsourcing, biotecnología, conservación de la biodiversidad

\section{CLASIFICACIÓN JEL}

\author{
L24, L65, Q57
}

\section{CONTENIDO}

Introducción, 1. Oportunidades para la implementación de esquemas de Knowledge Process Outsourcing (KPO); 2. KPO dentro del enfoque de cadena de valor propuesto en el DNP (2011); 3. TIC COL- USA y consolidación de esquemas de colaboración científica en la investigación aplicada que puedan involucrar esquemas de KPO, 4. Conclusiones, Bibliografía.

\footnotetext{
El presente artículo de reflexión hace parte de la línea de investigación sobre Políticas Públicas - Derecho de la Integración del Departamento de Derecho Económico de la Universidad Externado de Colombia. Su propósito es contribuir al debate actual sobre la liberalización de servicios en el marco de los tratados de libre comercio planteando posibles escenarios de interacción de las políticas en Ciencia Tecnología e innovación con del denominado biocomercio. No ha habido financiación de tipo público o privado para la elaboración del presente trabajo. Este trabajo fue presentado en el seminario "Deslocalización de servicios y cadenas globales de valor: ¿Nuevos factores de cambios estructurales en América Latina y el Caribe?", realizado en la ciudad de Santiago de Chile, el 18 y 19 de octubre de 2012.

** Abogado de la Universidad Externado de Colombia, especialista en Propiedad Intelectual de la misma Universidad y especialista en Gestión Pública e Instituciones Administrativas de la Universidad de los Andes. Master en Derecho Económico Internacional de la Universidad de Berna (Suiza). Investigador asociado del Departamento de Derecho Económico de la Universidad Externado de Colombia. Correspondencia: Departamento de Derecho Económico Universidad Externado de Colombia, Carrera 3 Este No. 9-96, primer piso, Bogotá, Colombia. correo electrónico: rodrigo.corredor@uexternado. edu.co.
} 


\title{
KNOWLEDGE PROCESS OUTSOURCING AS A KEY FOR COMPETITIVENESS OF COLOMBIAN BIOTECHNOLOGICAL SECTOR
}

\section{ABSTRACT}

Colombia as a country with mega diversity has important competitiveness advantages in the global market of raw materials and essential natural ingredients for a larger number of industrial activities.However, current practices related to provision of natural ingredients derived from biodiversity show divergences in relation to the scope of norms on technology transfer written in several multilateral instruments. This document tackles the concept of knowledge management outsourcing within the context of public policies oriented to the commercial use of biodiversity resources, searching for ideal conditions which may favor delocalization of research and development activities for multinational corporations related to bioprospection and basic research in such a way that biodiversity resources may be an object of aggregated value activities within the national territory.

\section{JEL CLASIFICATION}

L24, L65, Q57

\section{KEY WORDS}

Outsourcing, Biotechnology, Biodiversity Conservation

\section{CONTENTS}

Introduction 1. Opportunities for implementing KPO Schemes 2. KPO in the value's chain approach proposed in the DNP (2011) 3. Col-Usa TIC and consolidation of scientific collaboration Schemes in applied research which may involve KPO schemes 4. Conclusions; Bibliography.

\section{EXTERNALIZAÇÃO DA GESTÃO DO CONHECIMENTO COMO CHAVE PARA A COMPETITIVIDADE DO SETOR BIOTECNOLÓGICO COLOMBIANO.}

\section{RESUMO}

Na Colômbia como país muito diverso conta com importantes vantagens competitivas no mercado global de matérias-primas e ingredientes naturais essenciais para uma miríade de atividades industriais. No entanto, as práticas atuais relacionadas com o fornecimento de ingredientes naturais derivados da biodiversidade que apresentam diferenças no âmbito de aplicação das regras relativas à transferência de tecnologia contidas em vários instrumentos multilaterais. O presente documento, lida com o conceito de terceirização da gestão do conhecimento no contexto das políticas públicas que são direcionadas para a utilização comercial dos recursos da biodiversidade, procurando as condições ideais que podem favorecer a relocalização de atividades de investigação e desenvolvimento por empresas multinacionais como a bio-prospecção e a investigação de base, por isso, desta forma os recursos da biodiversidade podem ser objeto de atividades de valor agregado dentro do território nacional.

\section{PALAVRAS-CHAVE}

Outsourcing, biotecnologia, biodiversidade conservação.

\section{CLASSIFICAÇÃO JEL}

\author{
L24, L65, Q57
}

\section{CONTEÚDO}

Introdução, 1. As oportunidades para a implantação de esquemas de KPO; 2. KPO dentro da cadeia de valor proposto no DNP (2011); 3. CPT Col- EUA e consolidação dos diagramas de colaboração científica na investigação aplicada que pode envolver esquemas de KPO, 4. Conclusões Bibliografia. 


\section{INTRODUCCIÓN}

La economía colombiana se caracteriza por una amplia diversificación de su sector productivo. Profundizar este importante rasgo ha sido uno de los propósitos principales que ha motivado la reciente adopción de políticas públicas dirigidas a favorecer nuevos sectores productivos intensivos en capital y en conocimiento que permitan absorber buena parte de la mano de obra calificada en la transformación de recursos específicos ubicados en el territorio nacional.

Tal es el caso del sector biotecnológico, donde Colombia, en razón de su carácter megadiverso, cuenta con el potencial de lograr una ventaja competitiva ofreciendo a empresas multinacionales de este sector una reducción en cuanto a costos de investigación y desarrollo (IED) y/o generación de productos finales. En este sentido, el documento tiene como objetivo evaluar la pertinencia y oportunidad de los instrumentos de política pública propuestos recientemente, partiendo de la premisa según la cual la inversión en ciencia y tecnología constituye el insumo principal de la nueva economía del conocimiento.

Con el fin de orientar la discusión hacia el futuro, el análisis incluye una discusión sobre cómo el marco legal y regulatorio establecido en los capítulos de inversión y propiedad intelectual del Tratado de Libre Comercio Colombia-Estados Unidos (MCIT, 2015), pueden favorecen esquemas de Knowledge Process Outsourcing (KPO) en un sector considerado estratégico para la economía colombiana como lo es el de la biotecnología.

La primera parte identifica oportunidades para la implementación de esquemas de KPO en el marco de los programas de biocomercio. Una segunda parte aborda la KPO dentro del enfoque de cadena de valor propuesto en la política pública de uso sostenible de biodiversidad (DNP, 2011) mientras que el análisis final contenido en la tercera parte se focaliza en la incidencia que las normas contenidas en el TLC COL-USA (MCIT, 2015) pueden llegar a tener en la consolidación de esquemas de colaboración científica en investigación aplicada y cómo eventualmente en el marco de dicha cooperación podría favorecerse la deslocalización de algunas actividades de IED por parte de Corporaciones Multinacionales (CMN).

\section{OPORTUNIDADES PARA LA IMPLEMENTACIÓN DE ESQUEMAS DE KPO EN EL MARCO DE PROGRAMAS DE BIOCOMERCIO}

Para enmarcar la discusión en torno a la deslocalización de servicios asociados a la IED asociados a la investigación científica de los recursos de la biodiversidad, es necesario aclarar que la mayor parte de las discusiones en torno a este tópico 
suelen enmarcarse dentro de cuestiones más profundas relacionadas con la transferencia de tecnología y, de manera puntual, en relación con la instrumentalización de los objetivos contenidos en acuerdos multilaterales tales como los ADPIC ${ }^{1}$ o el reciente protocolo de Nagoya en el marco de las discusiones del Convenio sobre la Diversidad Biológica (CDB, 1992) ${ }^{2}$.

En dicho contexto son frecuentes las menciones en torno al problema de la ausencia de incentivos para concretar la trasferencia de tecnología hacia los países que albergan la mayor parte de la biodiversidad biológica del planeta.

De manera general, el problema fundamental de la agregación de valor respecto de los recursos de la biodiversidad parece estar ligado al hecho de que el proceso de transformación de un insumo o ingrediente natural tiende a localizarse en función de la estructura de costos definida por el empresario (el cual no necesariamente coincide con el lugar de extracción del ingrediente natural). Desde esta perspectiva, factores tales como la estabilidad macroeconómica, la seguridad jurídica, el sistema educativo, la proximidad a instituciones de referencia científica o simplemente la disposición de los entes reguladores entran a jugar un papel determinante en el análisis de costos de las empresas interesadas en la explotación comercial de estos recursos.

Colombia ha venido emprendiendo acciones en varios de estos frentes con el fin de atraer la inversión requerida para el desarrollo de sector industrial biotecnológico 3 .

No obstante, a presente, buena parte de las actividades en dicho sector pueden catalogarse como extractivas pero cualificadas en el sentido de que están sujetas al cumplimiento de estándares voluntarios, tal y como se viene dando en la indus -

$1 \quad$ ADPIC: Acuerdo sobre los aspectos de los Derechos de Propiedad Intelectual relacionados con el Comercio.

2 Convenio sobre la Diversidad Biológica CDB (1992), Protocolo de Nagoya, "Articulo 23, "De conformidad con los artículos 15, 16, 18 y 19 del Convenio, las Partes colaborarán y cooperarán en programas de investigación técnica y científica y desarrollo, incluyendo actividades de investigación biotecnológica, como un medio para lograr el objetivo de este Protocolo. Las Partes procurarán promover y alentar el acceso a la tecnología por las Partes que son países en desarrollo, en particular los países menos adelantados y los pequeños Estados insulares en desarrollo entre ellos y las Partes con economías en transición, y la transferencia de tecnología a estos, a fin de permitir el desarrollo y fortalecimiento de una base tecnológica y científica sólida y viable para lograr los objetivos del Convenio y el presente Protocolo. Cuando resulte posible y apropiado, dichas actividades de colaboración se llevarán a cabo en una Parte o las Partes, y con una Parte o las Partes, que proporcionan recursos genéticos que es o son el país o los países de origen de tales recursos, o una Parte o Partes que hayan adquirido los recursos genéticos de conformidad con el Convenir".

3 La declaración de exequibilidad de Ley de Protección de Datos Personales, por parte de la Corte Constitucional (sentencia C-748 de 2011), pone de presente el interés del Gobierno por abrir nuevos espacios a la inversión extranjera directa. Con la implementación de este cuerpo normativo Colombia se convierte en 'Puerto Seguro' para el desarrollo de esquemas de BPO y KPO. 
Externalización de la gestión del conocimiento como clave para la competitividad del sector biotecnológico colombiano

tria de los ingredientes naturales y más puntualmente dentro de los programas de biocomercio que se implementan en varios de los países de la cuenca amazónica4.

Estos emprendimientos distan mucho del proceso de agregación de valor del recurso genético mediante actividades científicas de investigación las cuales actualmente tienden a estar concentradas en los países industrializados.

\subsection{Naturaleza de los programas de biocomercio}

La filosofía y objeto de estos programas ha sido claramente condensada por Dutfield, (2000, p. 100); según el autor la iniciativa biocomercio apoyada por la Conferencia de las Naciones Unidas para el Comercio y el Desarrollo (UNCTAD) y el CDB (1992) se auto promueve como:

[...] una nueva aproximación a la conservación y uso sostenible de la biodiversidad. Para que los países en desarrollo puedan beneficiarse del creciente interés del sector privado en desplegar actividades de bioprospección; es necesario el establecimiento de condiciones de eficiencia y equidad que permitan superar obstáculos tales como: i. La propiedad sobre los recursos biológicos que no están bien definidos o que no pueden ser fácilmente protegidos; ii. Insuficiente información acerca de los usos actuales o potenciales de tales recursos; iii. Altos costos de transacción; iv. Ausencia de recursos técnicos o empresariales. De esta manera la estrategia promueve instrumentos económicos como medio para adicionar valor a los recursos biológicos, facilitar la apropiada transferencia de tecnología y mejorar la capacidad exportadora de estos países respecto de sus recursos.

En el ámbito nacional, la ausencia de datos que permitan medir la cantidad y calidad de la transferencia de tecnología que hasta hoy se ha logrado en el marco de estos programas dificulta hacer una estimación de hasta qué punto esquemas de KPO han podido ser implementados o podrían abrirse campo como alternativa para la agregación de valor de los recursos de la biodiversidad ${ }^{5}$.

\subsection{Cadenas de valor, biocomercio y KPO}

El enfoque de la política pública en materia de desarrollo comercial de la biotecnología en Colombia busca integrar a los agentes económicos locales en la cadena de

4 De acuerdo con (UNCTAD, 2012b) "the activities promoted by BioTrade tend to generate as much as possible benefits to local communities. The social impact in many cases involves the establishment of business linkages between the company and its suppliers (producers), thus reducing the length of the value chain by reducing unnecessary intermediaries. These linkages are not a "one-time" transaction but a continued and mutually beneficial commercial relationship and partnership. These include a fair, equitable and mutually agreed price, as well as capacity building, technology transfer, generation of social funds, among other benefits so that the direct producers and the community can also benefit".

5 De acuerdo con UNCTAD (2012a) la Iniciativa Biotrade cuenta con un BTIAS (BioTrade Impact Assessment System) por sus sigla en inglés, es de destacar el hecho de que dicho sistema no incluye ningún indicador o factor de medición en relación con el volumen o características cualitativas de la transferencia de tecnología obtenida en el marco de la ejecución de los programas de biocomercio. 
valor global de los productos biotecnológicos con el fin de derivar de tal interacción una serie de efectos dinámicos para la economía, principalmente en materia de empleo altamente calificado, mejoras en la productividad e impulso a la innovación. De acuerdo con el DNP (2011):

Actualmente el Ministerio de Ambiente, Vivienda y Desarrollo Territorial, se encuentra liderando la definición y formulación del Programa Nacional de Bio comercio Sostenible como parte de la revisión y actualización del Plan Estratégico Nacional de Mercados Verdes (...) Dicho Programa propone un enfoque de cadena de valor que desarrolle alianzas verticales o redes estratégicas entre un número de organizaciones o empresas independientes, que estén basadas en la confianza mutua. Bajo un enfoque de manejo compartido, donde la capacidad de gestión de la biodiversidad y los sistemas de conocimiento de las comunidades locales sean reconocidos formalmente por las autoridades ambientales y otros actores de las cadenas de valor, mejorando la gobernanza en torno a los recursos naturales al incluir a las comunidades en los procesos de definición de objetivos y toma de decisiones sobre el desarrollo de la cadena de valor y sobre la gestión de la biodiversidad.

Pero igualmente y de manera sorpresiva el mismo documento hace referencia a la creación por parte del Ministerio de Ambiente, Vivienda y Desarrollo Territorial de una:

[...] cadena de los Recursos Genéticos para el Uso Sostenible de la biodiversidad animal, vegetal y de microorganismos de interés para el sector agropecuario, que permitan un desarrollo de las bioindustrias y de la actividad agroindustrial, la cual buscará que el suministro de materias primas de la biodiversidad sea permanente y estable, ajustará los instrumentos sectoriales para que sean compatibles con el uso sostenible con fines comerciales de los recursos genéticos y el desarrollo de la biotecnología para la agroindustria y la agricultura empresarial, y se articulará con la oferta institucional de otros sectores para avanzar en la cadena de agregación de valor.

Tomando en cuenta lo anterior, se podría pensar que más que un uso estratégico de la biodiversidad, mediante la agregación de valor por medio I+D, tanto básica como aplicada, la prioridad de esta política pública es la de commoditizar de los recursos de la biodiversidad para facilitar su consecuente exportación a los mercados internacionales que demandan grandes volúmenes de nuevos ingredientes naturales ${ }^{6}$.

Ahora bien, no se pretende desconocer el hecho cierto de que el aprovechamiento comercial y sostenible de los recursos de la biodiversidad, así como su inclusión en la cadena global de valor, supone de suyo impactos positivos en las condiciones

6 En este sentido se considera relevante la reflexión efectuada por Martínez (2009, p. 179) en el sentido de que "... the consideration of of the physical and economic effects of trade and investment cannot simply bes set aside. If trade and investment regimes support and create incentives to certain economic and environmental relations, those have to be understood. (...), most exports of raw materials and natural resources have free or reduced tariff access to markets of industrialized countries, and one of the cornerstones of the FTAA and trade agreements of the United States is the deregulation of energy and natural resources sectors in Latin America. This shows the importance of trade regimes in orienting economic sectors affecting the environment." 
Externalización de la gestión del conocimiento como clave para la competitividad del sector biotecnológico colombiano

de vida y en la interacción de estos agentes con sus ecosistemas. No obstante, la existencia en el plano multilateral -e inclusive en el nivel de acuerdos comerciales bilaterales- de principios y objetivos que propenden por una transferencia efectiva de tecnología hacia los países que albergan la diversidad biológica es un factor relevante que debe orientar con rigurosidad el diseño de las políticas públicas hacia el cumplimiento de tales fines.

De allí la relevancia de analizar el concepto KPO en el contexto de la cadena global de valor del mercado de las modernas biotecnologías ${ }^{7}$, como un elemento más a tener en cuenta en la labor de reconducir las discusiones en el ámbito local sobre las políticas públicas y los mecanismos de implementación que se requieren para concretar el aprovechamiento de la biodiversidad, alejado de esquemas extractivos y promoviendo la consolidación de sectores de base tecnológica a partir de la comprensión de la lógica actual del mercado.

\section{KPO DENTRO DEL ENFOQUE DE CADENA DE VALOR PROPUESTO EN EL DNP (2011)}

Como ya se planteó, el DNP (2011) clarifica los instrumentos de política pública con los cuales el Estado se propone potenciar el desarrollo comercial de la biotecnología a partir del uso sostenible de la biodiversidad. El documento advierte de manera concreta sobre las oportunidades de Colombia en el panorama de la biotecnología en la arena global, caracterizado por un alto grado de concentración y el uso intensivo de factores de producción principalmente capital y mano de obra calificada.

Sin embargo, todo parece indicar que la externalización hacia Colombia de servicios asociados a I+D ha sido un campo desestimado por los técnicos encargados del diseño de esta política pública. Varias razones pueden explicar esta postura.

\subsection{Factor regulatorio}

En primer lugar, es necesario precisar que la ausencia de un estándar internacional que reconozca el valor estratégico de los recursos genéticos para los países en desarrollo influye negativamente en la decisión de las CMN de deslocalizar una parte de sus actividades de caracterización o investigación básica en el marco de

7 De acuerdo con la OECD (2010) "Two types of business have dominated biotechnology since 1970s - small and medium enterprises(SMEs) that concentrate on biotechnology research (commonly referred as "dedicated" biotechnology firms or DBFs) and large, vertically integrated firms (...)the value added chain for biotechnology extends from basic research to end-consumer (...)Many DBFs require years or decades to develop a discovery into a marketable product and lack of resources to manufacture, distribute and market their inventions. Their business model depends on obtaining financing from venture capital firms, an initial public offering (IPO) on the stock market, selling licenses to specialized knowledge to large firms under contract or as part of a joint venture."

8 Así como de los conocimientos tradicionales asociados a tales recursos. 
acuerdos de colaboración científica que propicien una efectiva transferencia de tecnología y que puedan generar el efecto derrame deseado en materia de promoción de ciencia, tecnología e innovación.

Ante esta realidad, las actividades de bioprospección en Colombia se adelantan dentro de un marco legal que, aunque bien concebido, no ha logrado el efectivo control del tránsito de recursos genéticos y biológicos dentro y fuera del territorio. Este factor, aparentemente desconectado del comercio en servicios, tiene una repercusión importante en la medida en que afecta la predictibilidad y seguridad jurídica que las empresas multinacionales demandan para adelantar proyectos de investigación dentro del territorio colombiano.

Por otro lado, la actitud poco clara por parte del Gobierno para concretar la implementación de normas en materia de acceso a recursos genéticos, así como la indecisión de apoyar un estándar más exigente en el plano multilateral, está llevando a que en la actualidad las actividades de bioprospección se adelanten de manera oportunista por parte de los agentes del mercado.

\subsection{Desconocimiento aparente de la racionalidad económica que justificaría esquemas de $\mathrm{KPO}$ en $\mathrm{I}+\mathrm{D}$}

Para entrar a analizar los impactos positivos que se pueden derivar de la implementación de esquemas de KPO aplicados al ciclo de innovación en materia de aprovechamiento de los recursos de la biodiversidad, es necesario partir de la importancia que para los países industrializados y sus CMN representa la accesibilidad a productos naturales (ingredientes) que no poseen en sus territorios, o que, aun poseyéndolos, se hace preferible importarlos como una manera de reducir los costos de producción.

Por otro lado, en el contexto de la sociedad de conocimiento es posible que la motivación para implementar un esquema de KPO pueda también estar fundamentada en el aprovechamiento del capital humano (existencia mano de obra calificada) que haga atractivo para estas empresas trasladar parte de sus actividades de IED y de esta manera reducir sus costos sin afectar la calidad de la investigación que se requiere para asegurar o mantener los niveles deseados de innovación.

Propiciar que el factor humano sea el determinante para la deslocalización de servicios de IED requiere de la implementación de políticas inclusivas en materia de educación, ciencia, tecnología e innovación, las cuales no son de corto plazo9. En

9 Kalmanovitz, (2007, p. 60) se refiere a las similitudes entre las economías colombiana y de Corea del Sur durante el periodo que va de 1950 a 2000, haciendo énfasis en cómo la implementación de políticas 
Externalización de la gestión del conocimiento como clave para la competitividad del sector biotecnológico colombiano

Colombia el DNP (2009) ${ }^{10}$ contiene las bases sobre las cuales la política en materia de desarrollo comercial de la biodiversidad plantea el cumplimiento de sus fines mediante una acción coordinada con el denominado Sistema Nacional de Ciencia, Tecnología e Innovación.

\subsubsection{Protección de empleos altamente calificados en los países industrializados como consecuencia de la crisis económica}

Una vez aclarada la existencia e interacción entre los dos instrumentos de política pública, pertinentes para el desarrollo comercial de la biodiversidad, resulta necesario indagar por aquellos factores exógenos que afectan la decisión de las CMN para emprender la deslocalización de estos servicios dando preponderancia a la IED como medio para absorber la mano de obra calificada en los países que alojan los recursos de la biodiversidad.

Una primera respuesta se puede ubicar en la tendencia internacional hacia la concertación de las actividades que involucran IED, la cual parecería consolidarse como consecuencia de la actual crisis económica. En este escenario cobra validez la observación efectuada ya hace algunos años por Correa, (2001, p. 261) en el sentido de que:

Large firms are decentralizing some of those activities to foreign subsidiaries and branches mainly in other OECD countries (...) The apparent 'globalization' of RED activities has created high expectations as to the transfer of RED capabilities to developing countries. Unfortunately, they are not justified. Large firms are decentralizing part of their R\&D activities in foreign countries, but R\&D is less internationalized than all other dimensions of corporate activity, such as production and sourcing. Overall, foreign ownership is either not significantly o risk negatively correlated to RED performance.

De tal manera que el reto actual de los gobiernos está en continuar con el proceso de promoción y consolidación a los incentivos en materia de ciencia y tecnología de manera tal que sus sistemas de innovación resulten atractivos para las empresas multinacionales cuando los efectos de la actual crisis económica se extingan.

públicas de corte intervencionista lograron resultados disímiles para los dos países. En tal sentido afirma: "El legado institucional de Corea del Sur fue el corporativismo japonés, del que copió los chaebols, apalancados por bancos propios. La reforma agraria distribuyo la tierra y contribuyo a abaratar los alimentos y las materias primas. Los altos impuestos permitieron financiar la universalización de la educación, sobre todo de la tecnológica"

10 Documento Conpes 3582, 27 de abril de 2009. Política Pública de Ciencia, tecnología e Innovación. 


\subsubsection{Efecto sobre el precio de los ingredientes naturales}

Otro elemento importante a tener en cuenta es el efecto que en la actualidad se deriva de la comoditización de estos ingredientes naturales derivados de la biodiversidad. En efecto, el precio relativamente bajo de estas materia primas seguirá sirviendo como incentivo negativo a la transferencia de tecnología, haciendo persistir la situación actual en la cual, empleos de baja calificación producen productos con bajo valor agregado pero que son remunerados a un precio satisfactorio para las comunidades que los producen.

Un escenario inverso, en el cual opere una reducción del precio como consecuencia de la deslocalización de actividades de IED a los países de origen puede presionar un aumento de los precios internacionales de algunos ingredientes naturales, afectando, en el corto plazo, a las comunidades que hoy en día derivan un beneficio por su participación en tales esquemas. Sin embargo, en el largo plazo la situación para el conjunto de la economía de los países megadiversos sería benéfica por cuanto la deslocalización de actividades relacionadas con la IED y/o producción involucraría la inclusión de mano de obra calificada dentro de la cadena de valor global.

\subsubsection{La preservación del statu quo en materia de biocomercio favorece en el corto plazo a las CMN así como a los intermediarios de estos programas}

Es claro que los actuales intermediarios asociados a programas de biocomercio no consideran conveniente para sus intereses la deslocalización de actividades de I+D hacia los países proveedores por cuanto su actividad económica principal perdería sentido.

\section{TLC COL-USA Y CONSOLIDACIÓN DE ESQUEMAS DE COLABORACIÓN CIENTÍFICA EN INVESTIGACIÓN APLICADA QUE PUEDA INVOLUCRAR ESQUEMAS DE KPO}

Múltiples factores juegan a favor de la deslocalización de actividades de alto valor agregado hacia los países en desarrollo; Juma y Konde (2005) plantean algunos de los factores que pueden mejorar la participación de los países en desarrollo en el mercado de las biotecnologías.

Although scientific advances in biotechnology appear to be concentrated in a small number of industrialized countries, there are various factors that would allow for the wider participation of developing countries in the new bioeconomy:

the growing recognition that the current patterns of globalization are untenable if they do not increasingly include developing country products. These countries depend on industries that are based on natural resources and therefore can benefit from the use of modern biotechnology; 
Externalización de la gestión del conocimiento como clave para la competitividad del sector biotecnológico colombiano

the fact that many of the techniques used in biotechnology research are becoming readily available because of scientific familiarity and are therefore relatively easy to acquire through sustained capacity development and enterprise development efforts; and

the fact that much of the initial research and development (RED) expenditure has already been borne by industrialized countries and what are needed are effective international technology partnerships.

En este sentido, tal y como se ha mencionado anteriormente en este documento, la dinámica actual de la investigación científica promueve la cooperación científica como principal motor para lograr el desarrollo económico y, al parecer, es en esta lógica que las disposiciones en materia de inversión ${ }^{11}$ y de propiedad intelectual ${ }^{12}$ contenidas en el marco del Tratado de Libre Comercio Colombia-Estados Unidos (MCIT, 2015) pueden servir de incentivo para que las CMN de dicho país emprendan procesos deslocalización de actividades de IED hacia el territorio colombiano.

Estas disposiciones son relevantes en la medida en que aclaran el alcance esperado en materia de transferencia de tecnología, la cual, desde el punto de vista de la protección a las inversiones, no podrá obedecer a una imposición o estar supeditada a condición alguna por parte del país receptor de la inversión, lo cual guarda absoluta concordancia con las normas establecidas en el acuerdo Trade Related Investments Measures (TRIMS), administrado por la Organización Mundial del Comercio, respecto de la prohibición de requisitos de desempeño.

Desde la perspectiva planteada por el capítulo de propiedad intelectual, los esquemas la deslocalización de actividades de alto valor agregado y su consecuente transferencia de tecnología parecen quedar supeditados al establecimiento de relaciones de cooperación científica, las cuales, a su vez, dependerán en buena

11 El capítulo relativo a inversiones dispone en su artículo 10.9 "1. Ninguna Parte podrá, en relación con el establecimiento, adquisición, expansión, administración, conducción, operación, venta u otra disposición de una inversión de un inversionista de una Parte o de un país que no sea Parte en su territorio, imponer ni hacer cumplir cualquier requisito o hacer cumplir cualquier obligación o compromiso de:

(...)

(f) transferir a una persona en su territorio una tecnología particular, un proceso productivo u otro conocimiento de su propiedad."

12 El capítulo relativo a Propiedad Intelectual establece en el artículo 16.12.:

"Promoción de la Innovación y el Desarrollo Tecnológico.

"1. Las Partes reconocen la importancia de promover la innovación tecnológica, la difusión de información tecnológica y el fortalecimiento de capacidades tecnológicas, incluyendo, según sea pertinente, proyectos de investigación conjuntos entre las Partes. Por lo tanto, las Partes buscarán y fomentarán oportunidades para la cooperación en ciencia y tecnología e identificarán áreas para dicha cooperación, y, según sea apropiado, realizar proyectos de colaboración de investigación científica" (negrilla fuera de texto).

" 2. Las Partes darán prioridad a la colaboración para avanzar en objetivos comunes en ciencia, tecnología e innovación y en apoyar asociaciones entre las instituciones de investigación públicas y privadas y la industria. Cualquiera de estas actividades de colaboración o transferencia de tecnología deberá estar basada en términos mutuamente acordados". 
parte del éxito de la implementación de las políticas públicas en materia de ciencia, tecnología e innovación ${ }^{13}$.

Es aquí donde tal vez los esquemas de KPO puedan constituirse en un aporte para la creación de la capacidad técnica requerida respecto de servicios específicos tales como la vigilancia tecnológica, el soporte en materia de licenciamiento de tecnologías propietario, la gestión de los derechos de propiedad intelectual respecto de los resultados de las investigaciones, y por último, la asesoría en materia de transferencia de tecnología, las cuales pueden ser determinantes de una inversión en ciencia y tecnología eficiente, flexible y orientada hacia resultados.

Es claro que los esquemas de KPO van a demandar un esfuerzo adicional para los países en desarrollo. La labor de atraer la Inversión Extranjera Directa (IED) hacia sectores intensivos en conocimiento supone un desarrollo avanzado de las competencias empresariales y de control sobre las actividades de sus empresas subordinadas. En este contexto es pertinente la observación hecha por Myro, y otros (2007. p. 86) respecto a cómo:

[...] algunos estudios muy recientes indagan en los determinantes de la elección entre las estrategias internacionales de subcontratación (offshoring outsourcing) y las de creación de filiales propias (offshoring insourcing), señalando que cuanto más intensivas en capital y en I+D son las producciones más tiende a prevalecer la segunda de las alternativas, pues resulta más difícil monitorizar los procesos de subcontratación internacional. Además, también se hace preferible esta opción en los países menos desarrollados, en los que resulta más difícil que un subcontratista se provea de los medios de capital necesarios para desarrollar la actividad.

Por último, y teniendo en cuenta el amplio rango de actividades implícitas en la IED en biotecnología, es probable que las CMN hagan depender la implementación de esquemas de KPO de la existencia de un determinado nivel de infraestructura, la cual en el caso colombiano dependerá de la capacidad de gestión de las instituciones públicas de investigación para la creación de un entorno tecnológico que favorezca todas las actividades de la cadena de valor que van desde la prospección hasta la aprobación de productos comercializables.

13 Con relación a la posibilidad efectiva de cooperación científica es necesario tomar en consideración lo expresado por Juma y Konde (2005 p. 13) para quienes :"Partnering activities are naturally more concentrated in the industrialized countries, but these agreements are being extended to developing countries, especially in agricultural biotechnology(...) partnering agreements could also play a key role in the development of technological capabilities in the firms and institutions in developing countries." 


\section{CONCLUSIONES}

En el caso colombiano existe evidencia de un conjunto de políticas públicas que pueden propiciar la implementación de esquemas de KPO, en el sector biotecnológico. Sin embargo, esto dependerá de la forma como las economías industrializadas asuman medidas de protección de los empleos calificados en este específico sector de la economía. El documento ha esbozado algunos sesgos en cuanto a la racionalidad económica. Superar tales sesgos implica un alto nivel de intervención de los entes estatales para que la IED, en el campo del aprovechamiento sostenible de la biodiversidad, pueda abrirse hacia nuevos esquemas de gestión que creen la necesidad de nuevos servicios.

Las disposiciones en materia de transferencia de tecnología, contenidas en el capítulo de propiedad intelectual del TLC COL-USA (MCIT, 2015), abren la posibilidad para la implementación de esquemas de KPO. Sin embargo, alcanzar este propósito dependerá de la forma como el Sistema Nacional de Ciencia Tecnología e Innovación logre vincular el uso sostenible de los recursos de la biodiversidad a esquemas de colaboración científica de manera tal que se valorice el potencial de los recursos genéticos y biológicos disponibles en las diferentes regiones del país.

Por otro lado, es indispensable que el programa biocomercio propicie las condiciones para que la efectiva transferencia de tecnología (y eventual implementación de esquemas de KPO) pueda concretarse en los países que albergan los recursos de la biodiversidad. Un buen punto de partida consistiría en orientar las metodologías de medición del impacto de estos programas para que también se incluyan indicadores de niveles de transferencia de tecnología o, por qué no, el nivel de deslocalización de las actividades de las empresas que utilizan ingredientes naturales con criterio de sostenibilidad

\section{BIBLIOGRAFÍA}

CDB-Convención sobre la Diversidad Biológica-(1992). Convenio sobre la Diversidad Biológica. [En Línea] Organización de Naciones Unidad.30p.

Correa, C. (2001). Emerging Trends: New Patterns of Technology Transfer. En: Patel, Surendra J., Roffe, P y Yusuf, A. International Technology Transfer: The Origins and Aftermath of the United Nations Negotiations on a Draft Code of Conduct, Londres, Kluwer Law International, 543p.

Dutfield, G., 2000, Intellectual Property Rights, Trade and Biodiversity. Londres, IUCN, Earthscan.

DNP-Departamento Nacional de Planeación (2009). Documento Conpes 3582. "Política Pública de Ciencia, Tecnología e Innovación'. Bogotá, 27 de abril, 68p. 
DNP-Departamento Nacional de Planeación (2011). Documento Conpes 3697. Política para el Desarrollo Comercial de la biodiversidad a partir del Uso Sostenible de la Biodiversidad. Bogotá, 14 de junio, 36p.

Juma, C. y Konde, V. (2005) Developing Countries in the global Bioeconomy: Emerging Issues. En: Trading in Genes: Development Perspectives on Biotechnology, Trade and Sustainability, Londres, Earthscan. 155p.

Kalmanovitz, S. (2007). Colombia en las dos fases de la globalización. En: Revista Economía Institucional, Vol. 9, No. 17, Bogotá:. Universidad Externado de Colombia. p. 43-74.

Myro, R.; Fernández, C.; Labrador, L.; Baides, A.; Álvarez, M. y Vega, J. (2007). Globalización y deslocalización. Importancia y efectos para la industria española, Madrid: Dirección General de la Política de la Pequeña y Mediana Empresa, Ministerio de Industria, Turismo y Comercio, 18p.

Martínez, D. (2009). Examination of the Environmental Cosequences of Trade Regimes in Latin America. En: Revista Observatorio de Políticas, Ejecución y Resultados de la Administración Pública, No. 9, Bogotá, Universidad Externado de Colombia, p.193-196.

MCIT-Ministerio de Comercio, Industria y Turismo- (2015). Acuerdo de Promoción Comercial entre la República de Colombia y Estados Unidos de América: Texto final del acuerdo. [En línea] [consultado el 05 de octubre de 2015].

OECD (2010). The Bioeconomy to 2030: Designing a policy agenda. Paris. 164p.

UNCTAD (2012b). Trade and Biodiversity: The BioTrade Experiences in LatinAmerica, Ginebra, Naciones Unidas, 57p.

UNCTAD (2012a) The BioTrade Impact Assessment System, Ginebra, consultado el 15 de agosto de 2013. Corte Constitucional (Sentencia C-748 de 2011) 